\title{
An ultra-low-molecular-weight heparin, fondaparinux, to treat retinal vein occlusion
}

\author{
Robert D Steigerwalt Jr. ${ }^{1, *}$, Antonella Pascarella ${ }^{2}$, Mauro De Angelis ${ }^{2}$, Francesco Ciucci ${ }^{3}$, \\ Francesco Gaudenzi ${ }^{4}$
}

${ }^{1}$ Via A. Brofferio 6, Rome, Italy;

${ }^{2}$ Medical Retina, Ophthalmic Hospital, Rome, Italy;

${ }^{3}$ Saint Peter's Hospital, Fatebenefratelli, Rome, Italy;

${ }^{4}$ Strada Panoramica Adriatica 112, Pesaro Italy.

\begin{abstract}
Summary Retinal vein occlusions may decrease visual acuity. There is no known therapy to treat ocular thrombosis. The authors used fondaparinux, an ultra-low-molecular-weight heparin, to treat 13 consecutive cases of recent-onset retinal vein occlusions. Two patients with renal insufficiency were not included. Eight central retinal vein occlusions and 5 branch retinal vein occlusions in 13 patients were treated with subcutaneous fondaparinux $2.5 \mathrm{mg}$ once a day. The patients were seen every 2 weeks. Macular edema was treated with intravitreal injections of anti-vascular endothelial growth factor or steroids. Two patients elected to discontinue treatment. Of the remaining 11,9 occlusions resolved in 1.5 to 13.5 months with rapid resolution of retinal edema and hemorrhage as soon as the occlusions resolved. One patient had a retinal vein that was still occluded after 8 months of therapy and 1 had retinal vein occlusion that partially resolved after 15 months of treatment. Of the 9 eyes with occlusions that resolved, visual acuity improved in 7 . In 2, visual acuity decreased due to macular ischemia. Occlusion recurred in 12.5 months after the suspension of initial treatment. This patient is again being treated with fondaparinux $2.5 \mathrm{mg}$. No hemorrhaging occurred. Fondaparinux $2.5 \mathrm{mg}$ can be given subcutaneously once a day to patients with recent-onset retinal vein occlusions without renal insufficiency. An occlusion may take a number of months to resolve. Once the vein occlusion has resolved, retinal edema and hemorrhage rapidly resolve and vision improves. Macular edema should be treated while waiting for the vein occlusion to resolve.
\end{abstract}

Keywords: Fondaparinux, ultra-low-molecular-weight heparin, low-molecular-weight heparin, central retinal vein occlusion, branch retinal vein occlusion

\section{Introduction}

Central retinal vein occlusion (CRVO) is the second most common vascular cause of the loss of visual acuity (VA) (1). The precise etiology is not known although different risk factors have been identified (2). Hayreh

Released online in J-STAGE as advance publication June 15, 2016.

*Address correspondence to:

Dr. Robert D Steigerwalt Jr., Via A. Brofferio 6, Rome, Italy 00195.

E-mail: r.steigerwalt@libero.it divided CRVOs into 2 categories: a nonischemic CRVO or "venous stasis retinopathy," and an ischemic CRVO or "hemorrhagic retinopathy" (3). A branch retinal vein occlusion (BRVO) involves one of the retinal vein branches and usually has less of an impact on vision. BRVOs have been divided into nonischemic, indeterminate, and ischemic forms depending on the amount of capillary non-perfusion (4). The current study used fluorescein angiography to assess capillary non-perfusion and the description by Magargal in order to categorize retinal vein occlusion (RVO) as ischemic or nonischemic. Minimal to moderate (less than 50\%) capillary non-perfusion was considered nonischemic 
while more than $50 \%$ capillary non-perfusion was considered ischemic (5).

Ophthalmologists currently treat an RVO primarily with injections of intravitreal anti-vascular endothelial growth factor (anti-VEGF) agents or intravitreal steroids along with laser treatment of ischemic areas when needed. These injections can resolve severe macular edema and temporarily improve VA while waiting for the venous blockage to spontaneously resolve through recanalization or collateral vessel formation. These injections are not known to help remove or resolve a thrombus $(6,7)$.

There are 3 types of heparins: unfractionated heparin (UFH), low-molecular-weight heparin (LMWH) such as enoxaparin (Clexane), and ultra-low-molecular-weight heparin (ULMWH) such as fondaparinux sodium (Arixtra). Due to their ability to bind to antithrombin III (AT-III), heparins have anticoagulant activity. ATIII undergoes a conformational change that allows it to interact with coagulant enzymes such as thrombin (factor IIa) and factor Xa. UFH functions well as an anticoagulant but adverse bleeding episodes can occur while UFH is used in antithrombotic therapy. This is due to its high antithrombin (anti-factor IIa) activity. UFH can also cause heparin-induced thrombocytopenia (HIT), a life-threatening complication, and allergic reactions. LMWHs, such as enoxaparin, also react with factor Xa but react much less with thrombin (factor IIa), which is why they are less apt to cause bleeding. Adverse bleeding, HIT, and interference with platelets can still occur with the use of enoxaparin. ULMWHs, such as fondaparinux sodium, have the highest anti-factor Xa activity and the lowest anti-factor IIa activity compared to UFH and LMWHs. This results in the lowest rate of bleeding among heparins and potent anticoagulant activity (Table 1) (8). Fondaparinux is a chemically synthesized pentasaccharide with highly potent antifactor Xa activity. Since this pentasaccharide is too short to bridge AT-III to thrombin, fondaparinux has little anti-factor IIa activity and thus a markedly reduced risk of causing hemorrhaging (Table 1). As with enoxaparin, fondaparinux is administered subcutaneously; since it has a half-life of $17-21 \mathrm{hr}$, it is given once a day. Fondaparinux is metabolized principally in the kidneys and is contraindicated in patients with renal impairment. One advantage of fondaparinux is that the dosage does not need to be adjusted for weight or age because of its pharmacokinetics, i.e. its specific binding to anti-thrombin and its almost $100 \%$ bioavailability. Fondaparinux is administered subcutaneously in a dose of $2.5 \mathrm{mg}$ once a day. Fondaparinux has several disadvantages. Unlike UFH, fondaparinux has no antidote. It has a long half-life and it accumulates in patients with renal insufficiency. Some immune mediators can affect the absorption, activity, and metabolism of fondaparinux (8).

As previously reported, enoxaparin was used in
Table 1. Comparison of the anti-Xa and anti-IIa activity of UF heparin, enoxaparin, and fondaparinux

\begin{tabular}{lcc}
\hline Items & Anti-Xa (U mg-1) & Anti-IIa (U mg-1) \\
\hline UF heparin & 193 & 193 \\
Enoxaparin & 105 & 27 \\
Fondaparinux & $805 \pm 27$ & $<0.1$ \\
\hline
\end{tabular}

IIa, factor IIa; Xa, factor Xa

ophthalmology to treat 7 cases of RVO and 1 case of an orbital vein occlusion (9). In all 8 cases of venous thrombosis, the thrombus resolved and vision improved in 6 of 8 cases. The treatment lasted from weeks to months. There was one episode of spontaneous bleeding in an arm muscle requiring surgical drainage that did not affect the positive outcome of orbital thrombosis treatment.

Since LMWHs can resolve RVOs and have a minimal risk of causing HIT and bleeding, and also require constant platelet monitoring, the current study sought to evaluate an ULMWH, fondaparinux, with a markedly decreased risk of hemorrhaging and no need for platelet monitoring, in the treatment of RVOs. Presented here are the results of the use of fondaparinux, together with intravitreal anti-VEGF agents or steroids, in the treatment of RVOs.

\section{Materials and Methods}

Since March 2012, the current authors have seen 15 consecutive patients with recent-onset RVOs. All of the patients had complete eye examinations including the Snellen VA test (best-corrected), a slitlamp examination, Goldmann tonometry, and a dilated fundus examination. They also underwent fluorescein angiography to verify the diagnosis of an occlusion and to evaluate the extent of retinal ischemia. Ocular coherent tomography (OCT) was done to measure macular edema and, when necessary, peripheral retinal edema. At around the same time, all of the patients were seen by an internist for a complete medical evaluation, an electrocardiogram (EKG), and routine blood work including kidney function tests. Two patients had mild renal failure and were excluded from this study. Once RVO was diagnosed and the medical evaluation was complete, the remaining 13 patients received a through explanation of their condition and the risks involved in the experimental use of fondaparinux to resolve the RVO. Written consent was obtained before beginning treatment.

Thirteen patients began treatment with fondaparinux $2.5 \mathrm{mg}$ a day. Two patients elected to discontinue the fondaparinux and seek treatment at other facilities, 1 after 5 months and 1 after 6 months of treatment. Dropouts were not due to complications. The remaining 11 patients continued treatment and have been followed to date. The patients were asked to avoid physical 
Table 2. Clinical characteristics of patients and treatment outcomes

\begin{tabular}{|c|c|c|c|c|c|c|c|c|c|c|}
\hline Pat & Age/sex & Type/I & Onset & Duration & $\mathrm{VAb}$ & VAa & $\mathrm{OCTb}$ & OCTa & Intravit & status \\
\hline 1 & $35 \mathrm{M}$ & CRVO & $1 d$ & $4.5 \mathrm{mo}$ & $2 / 10$ & $10 / 10$ & 473 & 205 & dexam & open \\
\hline 2 & $78 \mathrm{~F}$ & BRVO & $10 \mathrm{~d}$ & $3.5 \mathrm{mo}$ & $3 / 10$ & $9 / 10$ & 341 & 185 & bevac & open \\
\hline 3 & $78 \mathrm{M}$ & I-CRVO & $21 d$ & $4 \mathrm{mo}$ & $1 / 30$ & $1 / 30$ & 601 & 153 & aflib & open \\
\hline 4 & $45 \mathrm{~F}$ & I-CRVO & $2 d$ & $8 \mathrm{mo}$ & $1 / 10$ & $1 / 10$ & 705 & 261 & dexam & open \\
\hline 5 & $61 \mathrm{M}$ & CRVO & $1 d$ & $5.5 \mathrm{mo}$ & $10 / 10$ & $10 / 10$ & 260 & 223 & none & open \\
\hline 6 & $73 \mathrm{M}$ & CRVO & $1 \mathrm{mo}$ & $1.5 \mathrm{mo}$ & $5 / 10$ & $7 / 10$ & 524 & 194 & aflib & open \\
\hline 7 & $82 \mathrm{~F}$ & CRVO & $1.5 \mathrm{mo}$ & $2.5 \mathrm{mo}$ & $1 / 30$ & $4 / 10$ & 510 & 236 & dexam & open \\
\hline 8 & $74 \mathrm{~F}$ & BRVO & $35 d$ & $2 \mathrm{mo}$ & $1 / 30$ & $4 / 10$ & 491 & 163 & dexam & open \\
\hline $8 a$ & $74 \mathrm{~F}$ & BRVO & $14 d$ & & $1 / 30$ & & 995 & & dexam & closed again \\
\hline 9 & $66 \mathrm{~F}$ & BRVO & $21 d$ & $15 \mathrm{mo}$ & $8 / 10$ & $10 / 10$ & 310 & 213 & none & partially open \\
\hline 10 & $76 \mathrm{~F}$ & I-BRVO & NK & $6 \mathrm{mo}$ & $9 / 10$ & $10 / 10$ & 215 & 219 & none & not open \\
\hline 11 & $64 \mathrm{~F}$ & CRVO & $1 d$ & $13.5 \mathrm{mo}$ & $5 / 10$ & $8 / 10$ & 743 & 228 & none & open \\
\hline
\end{tabular}

Aflib, aflibercept; bevac, bevacizumab; BRVO, branch retinal vein occlusion; CRVO, central retinal vein occlusion; dexam, dexamethasone; Duration, duration of treatment with fondaparinux; I, ischemic; NK, not known; OCTb, macular thickness in microns (um) according to OCT before treatment; OCTa, macular thickness in um according to OCT after treatment; Onset, time between onset of venous occlusion and the initiation of treatment; VAb, visual acuity before treatment; VAa, visual acuity after treatment.

activity or aggressive sports that could lead to bleeding.

\section{Results and Discussion}

Patients consisted of 4 males and 7 females with an average age of $66.5 \mathrm{yr}$ and a range of $35-85 \mathrm{yr}$. There were 7 CRVOs and 4 BRVOs in these 11 patients. Of the 11 treated RVOs, 9 resolved. Of the remaining 2, 1 (Patient 9 in Table 2) resolved in the center of the macula but the periphery remained occluded with peripheral edema on OCT and clinical signs of an intraretinal hemorrhage and the other (Patient 10) did not change so peripheral blockage remained but there was no central edema. These 2 patients elected to continue treatment. Two of the CRVOs were ischemic and were treated with pan retinal argon laser photocoagulation, and 1 of the BRVOs was ischemic and was treated with sectoral retinal argon laser photocoagulation of the ischemic areas. The time from onset of the RVO to the beginning of therapy was 1-2 days in 4 patients, 10 days to 5 weeks in 6 patients, and unknown in 1 patient (Table 2)

Fondaparinux $2.5 \mathrm{mg}$ was subcutaneously administered once a day to all 13 of the original patients. There were no episodes of systemic or ocular bleeding. The patients underwent eye examinations, including a VA test and fundus examination, every 2 weeks while being treated. Of the 11 patients who continued treatment, 7 received an intravitreal injection of steroids or other anti-VEGF agent to treat macular edema. One of the 7 required a second injection after 4 months because of recurrent macular edema. The drug that was intravitreally injected depended on its availability and the physician's medical judgement. Four received a dexamethasone intravitreal implant (Ozurdex), 2 received 0.05 cc of aflibercept (Eylea), and 1 received $0.05 \mathrm{cc}$ of bevacizumab (Avastin). Three patients did not receive intravitreal injections because they did not have severe enough macular edema to justify an injection. One patient refused the injection (Patient 11). Arixtra was suspended $24 \mathrm{hr}$ before each injection and restarted the same day 1-2 hr after the intravitreal injection. In all 11 patients, the fundus was examined every 2 weeks to evaluate whether the blockage resolved or not. Since the intravitreal injections resolved macular edema, the fundus was examined to ascertain a decrease in intraretinal bleeding and improvement in VA as an indication that the blockage had resolved. If clinical signs of a blockage were absent, Arixtra was reduced to every other day for 2 weeks and then suspended while waiting for a second OCT and fluorescein angiography. OCT and fluorescein angiography were performed, albeit not always immediately, to determine if the blockage had resolved or not. The rapid resolution of intraretinal bleeding, absence of new macular edema, and improvement in VA were evident clinically and served as indicators that the blockage had resolved. Of the 2 patients in whom a retinal vein was still occluded, one (Patient 9) had macular edema that resolved after 11 mo of therapy but peripheral edema persisted and was followed with OCT. This same patient with an occluded retinal vein (Patient 9 in Table 2) weighed 120 kilos at the start of treatment and 100 kilos at 12 months. Two patients developed collateral vessels at the optic nerve head before the occlusions resolved while being treated with fondaparinux (one dropped out at 5 months and the other was Patient 6). One patient (Patient 8 ) with a BRVO had an occlusion that resolved after 2 months with visual improvement, resolution of intraretinal bleeding, and resolution of macular edema. The therapy was gradually suspended and the patient was started on aspirin once a day. After 2.5 months on aspirin, the patient (Patient 8a) suddenly developed vision loss. A new BRVO occurred in the same area and the same therapy with fondaparinux $2.5 \mathrm{mg}$ was started again. After 6 months of this treatment, the retinal vein remains occluded. 
Nine of the RVOs resolved, 2 remained occluded, and 1 of the 9 that had resolved later recurred. Of the occlusions that resolved, 3 resolved in 1.5 to 2.5 months and the others took longer, with 1 resolving after 13.5 months. In the occlusions that recurred, the blockage remained after 6 months of therapy (Table 2). The patients were examined every 2 weeks while being treated. When clinical signs of an occlusion were absent, fondaparinux $2.5 \mathrm{mg}$ was given every other day for 2 weeks and the patient was re-examined before stopping treatment. An OCT and fluorescein angiography were performed, albeit not always immediately, to verify that the occlusion resolved. The 2 patients who had occlusions that failed to resolve have BRVOs. One patient (Patient 9) had no macular edema but still had peripheral intraretinal bleeding and retinal edema after 15 months according to OCT and another (Patient 10) similarly had no macular edema but still had peripheral intraretinal bleeding and retinal edema after 7 months. Both patients elected to continue treatment. Macular edema improved in 1 patient (Patient 9) and, the patient elected to continue treatment because of amblyopia in the other eye.

Fondaparinux, an ULMWH, appears to be effective in resolving recent-onset RVOs and restoring VA without causing episodes of bleeding or requiring platelet monitoring. Nine patients out of the 11 treated had an occlusion that resolved, but the treatment took several months. One occulusion resolved after 13.5 mo. Of the 9 eyes with an occlusion, 7 had visual improvement and 2 with macular ischemia did not. Macular edema needs to be treated while waiting for an occlusion to resolve in order to restore VA. Macular edema can be treated with intravitreal steroids or injections of anti-VEGF agents. Anti-VEGF agents are not known to have anticoagulative effects in a vein occlusion but may have other benefits. Large amounts of VEGF are released in ischemic vein occlusions (10). This creates macular edema and anti-VEGF agents can be used to treat that edema $(6,7)$. However, studies in primates have indicated that VEGF induces capillary endothelial cell proliferation within blood vessels, and particularly in veins, leading to intussusceptions and endothelial cell wall bridging within venules in some areas and vascular closure and non-perfusion in others (11). Inhibition of VEGF in the eye should reduce or prevent VEGF-induced intraluminal capillary endothelial cell proliferation and thus prevent a VEGFinduced reduction in venous flow. This would prevent the continued propagation of venous ischemia after the original vein occlusion and decrease the need for collateral vessel formation. Thus, the current study administered anti-VEGF agents while waiting for the occlusions to resolve. Treating edema hampers the clinical evaluation of when an RVO has resolved. Regular fundus examinations must be performed to follow an intraretinal hemorrhage or the recurrence of macular edema in order to determine when an occlusion has resolved. OCT and fluorescein angiography were used to help decide when the occlusion resolved. Once the RVO resolved, the edema and intraretinal hemorrhage rapidly resolved. Once the occlusion has resolved, fondaparinux should be stopped gradually every other day for 2 weeks before its suspension and the injections of anti-VEGF agents can be suspended. The authors' experience has been that suddenly stopping enoxaparin occasionally results in recurrence of an RVO within weeks in about $5 \%$ of patients (unpublished data). Therefore, once an RVO had resolved enoxaparin was administered every other day for 2 weeks in order to evaluate the risk of occlusion recurring before enoxaparin was completely suspended. The same approach was used for fondaparinux. Fondaparinux $2.5 \mathrm{mg}$ was administered every other day for 2 weeks before it was completely stopped. When an RVO was treated with enoxaparin, occlusion recurred after its suspension. This occurred in Patient 82.5 months after fondaparinux was suspended and aspirin was given. In that patient, a blockage developed in the same area of the retina. Collateral vessels at the optic nerve head were noted in 2 patients. This is not surprising because the treatment to resolve an occlusion took several months in most of the current patients. Collateral vessel formation is a natural ocular response to blocked venous flow. The venous system takes a number of months to recover with fondaparinux and a natural ocular response can be expected in the interim.

Fondaparinux did not cause severe bleeding. UFH is an anticoagulant because of its effect on factor Xa but it has a high incidence of hemorrhaging because of its effect on factor IIa, in addition to its risk of HIT and platelet complications. Enoxaparin is an anticoagulant with a similar effect on factor Xa but a markedly reduced effect on factor IIa, with a much reduced incidence of hemorrhaging as well as HIT or platelet complications. Fondaparinux has a negligible effect on factor IIa, and therefore less of a risk of hemorrhaging, while having a potent effect on factor Xa. This means that fondaparinux has a potent anticoagulant effect in a venous occlusion (Table 1) (8). Platelet monitoring is not necessary, though it has been used in cases of HIT. Because of the bioavailability of fondaparinux, only 1 dose of $2.5 \mathrm{mg}$ is administered subcutaneously per day. Fondaparinux should not be administered to patients with renal insufficiency $(8)$.

The final VA may depend on the duration of macular edema and the presence of macular ischemia. It may also depend on the time from the onset of an occlusion to the beginning of treatment. In the current patients, the time from the onset of an occlusion to the beginning of treatment was 1 day to 5 weeks, though that period was not known in Patient 10. Improvement in VA and the extent to which an occlusion resolves may differ with a longer time before the initiation of treatment. 
In conclusion, fondaparinux appears to be effective at resolving recent-onset RVOs without causing hemorrhaging. Macular edema and retinal ischemia also need to be treated while waiting for the occlusion to resolve. Patients need to be seen frequently in order to determine when to suspend treatment.

\section{References}

1. Clarkson JG. Central retinal vein occlusion. In: Retina. 3rd ed, Vol 2. (Ryan SJ, ed.). Mosby, St Louis, MO, USA, 2001: pp. 1368.

2. Risk factors for central retinal vein occlusion. The Eye Disease Case-Control Study Group. Arch Ophthalmol. 1996;114:545-554.

3. Hayreh SS. Classification of central retinal vein occlusion. Ophthalmology. 1983; 90:458-74.

4. Magargal LE, Kimmel AS, Sanborn GE, Annesley WH Jr. Temporal branch retinal vein occlusion: a review. Ophthalmol Surg. 1986;17:240-246.

5. Magargal LE, Brown GC, Augsburger JJ, Parrish RK 2nd. Neovascular glaucoma following central retinal vein occlusion. Ophthalmology. 1981; 88:1095-1101.

6. Ferrara DA, Koizumi H, Spaide RF. Early bevacizumab treatment of central retinal vein occlusion. Am J
Ophthalmol. 2007; 144:864-871.

7. Tservakis I, Koutsandrea C, Papaconstantinou D, Paraskevopoulos T, Georgalas I. Safety and efficacy of dexamethasone intravitreal implant (Ozurdex) for the treatment of persistent macular edema secondary to retinal vein occlusion in eyes previously treated with antivascular endothelial growth factors. Curr Drug Saf. 2015; 10:145-151.

8. Liu Z, Ji S, Sheng J, Wang F. Pharmacological effects and clinical applications of ultra low molecular weight heparins. Drug Discov Ther. 2014; 8:1-10.

9. Steigerwalt RD Jr, Cesarone MR, Belcaro G, Quercioli P, Lofoco G, Ciucci F, Pascarella A, Angelis MD, Rapanetta L, Nebbioso M. Retinal and orbital venous occlusions treated with enoxaparin. J Ocul Pharmacol Ther. 2008; 24:421-426.

10. Pe'er J, Folberg R, Itin A, Gnessin H, Hemo I, Keshet E. Vascular endothelial growth factor upregulation in human central retinal vein occlusion. Ophthalmology. 1998; 105:412-416.

11. Tolentino MJ, McLeod DS, Taomoto M, Otsuji T, Adamis AP, Lutty GA. Pathologic features of vascular endothelial growth factor-induced retinopathy in the nonhuman primate. Am J Ophthalmol. 2002; 133:373-385.

(Received May 7, 2016; Revised May 30, 2016; Accepted June 2, 2016) 